\title{
Molecular scale electronics: syntheses and testing
}

\author{
William A Reinerth $\dagger$, LeRoy Jones II $\dagger$, Timothy P Burgin $\dagger$, \\ Chong-wu Zhou $\ddagger$, C J Muller $\ddagger$, M R Deshpande $\ddagger$, Mark A Reed $\ddagger$ \\ and James $M$ Tour $\dagger$
}

\author{
† Department of Chemistry and Biochemistry, University of South Carolina, \\ Columbia, SC 29208, USA \\ $\ddagger$ Department of Electrical Engineering, Yale University, PO Box 208284, New \\ Haven, CT 06520, USA
}

Received 17 March 1998

\begin{abstract}
This paper describes four significant breakthroughs in the syntheses and testing of molecular scale electronic devices. The 16-mer of oligo(2-dodecylphenylene ethynylene) was prepared on Merrifields resin using the iterative divergent/convergent approach which significantly streamlines the preparation of this molecular scale wire. The formation of self-assembled monolayers and multilayers on gold surfaces of rigid rod conjugated oligomers that have thiol, $\alpha, \omega$-dithiol, thioacetyl, or $\alpha, \omega$-dithioacetyl end groups have been studied. The direct observation of charge transport through molecules of benzene-1, 4-dithiol, which have been self-assembled onto two facing gold electrodes, has been achieved. Finally, we report initial studies into what effect varying the molecular alligator clip has on the molecule scale wire's conductivity.
\end{abstract}

Future computational systems are likely to consist of logic devices that are ultra dense, ultra fast, and molecularsized [1-3]. The slow step in existing computational architectures is not usually the switching time, but the time it takes for an electron to travel between devices. By using molecular scale electronic interconnects $\dagger$, the transmit times could be minimized, resulting in computational systems that operate at far greater speeds than is presently attainable from conventional patterned architectural arrays [1]. There is another technical advantage that might also be gained from molecular scale devices. A powerful computational system presently utilizes about $10^{10}$ siliconbased devices. If devices were to be based upon single molecules [3r], using routine chemical syntheses, one could prepare approximately $10^{23}$ devices in a single reaction flask. Of course, the task of addressing large arrays of ordered molecular scale devices is presently unattainable; however, the potential is certainly enough to maintain current and future interests.

Though it is well documented that bulk conjugated organic materials can be semiconducting or even conducting when doped [5], we have only recently deter-

\footnotetext{
$\dagger$ 'Molecular electronics' is a poorly defined term since some authors refer to it as any molecular-based system such as a film or a liquid crystalline array. Other authors, including us, prefer to reserve the term 'molecular electronics' for single molecule tasks, such as single moleculebased devices or single molecular wires. Due to this confusion, we have chosen here to follow the terminology of [4] by using two subcategories, namely 'molecular materials for electronics' for bulk applications and 'molecular scale electronics' for single molecule applications.
}

mined how thiol-ended rigid rod conjugated molecules orient themselves on gold surfaces [6], and how we could record electronic conduction through single undoped conjugated molecules that are end-bound onto a metal probe surface [7]. We have previously described the syntheses of soluble oligo(3-ethyl-2, 5-thiophene-ethynylene)s and oligo(2-alkyl-1, 4-phenylene-ethynylene)s, potential molecular scale wires, by a rapid, solution phase iterative divergent/convergent doubling approach $[8,9]$, as well as the syntheses and attachments of protected thiol moieties to one or both ends of the molecular scale wires. These thiols serve as molecular scale alligator clips for adhesion of the molecular scale wires to the gold probes $[6,7]$.

Herein we report four significant breakthroughs that could have dramatic implications in the syntheses and testing of molecular scale electronic devices. First, we have completed the synthesis of the dodecyl-containing 16-mer on Merrifield's resin (chloromethyl polystyrene) using the iterative divergent/convergent approach; a method which significantly streamlines the preparation [10]. Second, we have studied the formation of self-assembled monolayers (SAMs) and multilayers on gold surfaces of rigid rod conjugated oligomers that have thiol, $\alpha, \omega$ dithiol, thioacetyl, or $\alpha, \omega$-dithioacetyl end groups. Third, molecules of benzene-1, 4-dithiol have been self-assembled onto the two facing gold electrodes of a mechanically controllable break junction (MCB) to form a statically stable gold-sulfur-aryl-sulfur-gold system, which allows for direct observation of charge transport through the molecules. Finally, we examine what effect varying the 


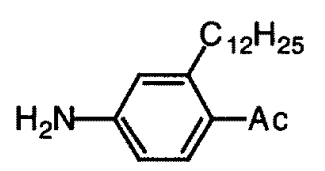

1. $F B U O N O, \mathrm{BF}_{3} \mathrm{Et}_{2} \mathrm{O}$

2. 2-(ethylamino)ethanol, $85 \%$

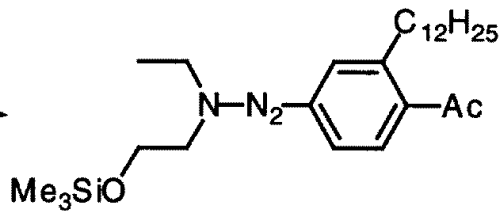

1. LDA

2. $\mathrm{CIPO}(\mathrm{OEt})_{2}$

3. $\mathrm{NEt}_{3}, \mathrm{Me}_{3} \mathrm{SiCl}, 95 \%$ $\mathrm{Me}_{3} \mathrm{SiO}^{\prime}$

3. LDA (2 equiv)

4. $\mathrm{Me}_{3} \mathrm{SiCl}$

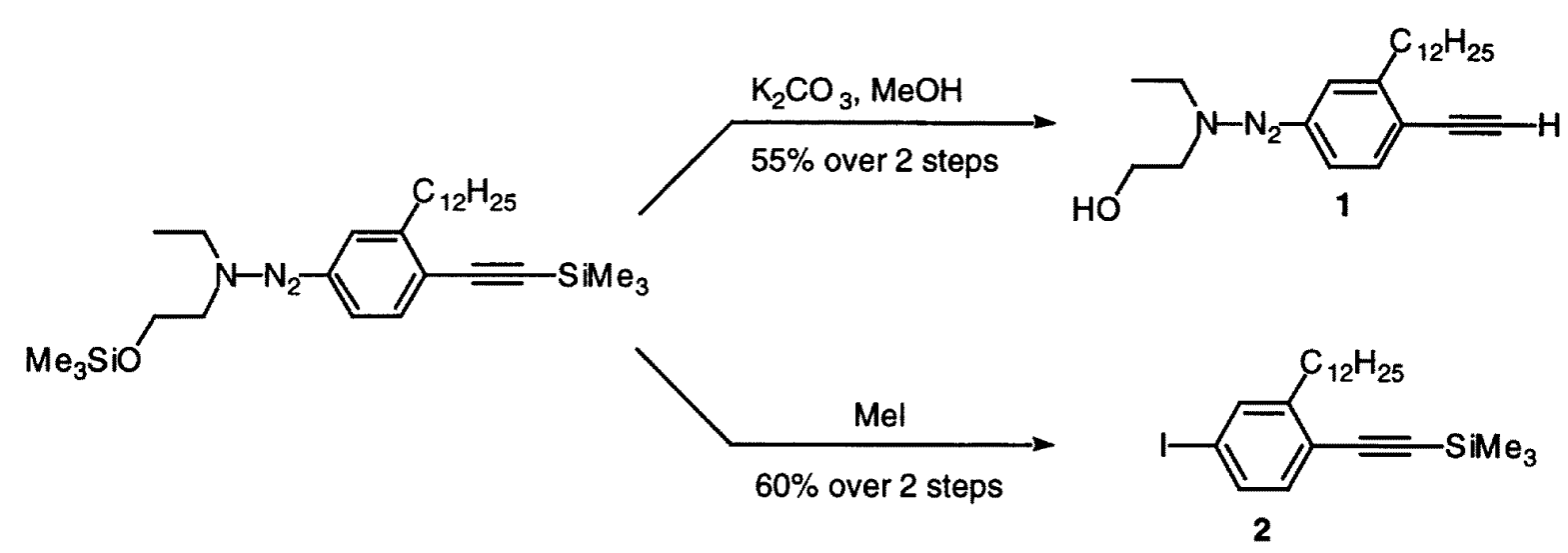

Scheme I. Synthesis of the dodecyl-containing anchor 1 and monomer 2.

molecular alligator clip has on the molecular scale wire's conductivity.

The monomers needed for the polymer support synthesis were prepared as depicted in scheme I. The silylated triazene was divided into two portions; the first portion was desilylated to form $\mathbf{1}$, the anchor unit to be attached to the polymer support, while the second portion was iodinated [10] to form the iodoarene 2. Attachment of $\mathbf{1}$ to the polymer support resin, and the oligomer syntheses on the polymer support are depicted in scheme II. This polymer supported approach greatly simplifies the isolation and purification of oligomers 5, 8, 11, and 14 .

As a prelude to the utilization of molecular scale wires in molecular scale electronic devices, it is necessary to understand the molecular ordering on metal surfaces. These sulfur-terminated conjugated oligomers form SAMs on gold surfaces by attachment of the thiol end groups which serve as molecular scale alligator clips [6a]. The SAMs were analyzed using ellipsometry, x-ray photoelectron spectroscopy (XPS), and infrared external reflectance spectroscopy. The thiol moieties usually dominate adsorption on the gold sites; interactions with the conjugated $\pi$-systems are weaker. Rigid $\operatorname{rod} \alpha, \omega$ dithiols form assemblies in which one thiol group binds to the surface while the second thiol moiety projects upward at the exposed surface of the SAM. In situ deprotection of the thiol moieties by deacylation of thioacetyl groups using $\mathrm{NH}_{4} \mathrm{OH}$ permits the formation of SAMs without the need to isolate the oxidatively unstable free thiols. Moreover, direct adsorption, without exogenous base, of the thioacetyl-terminated oligomers can be accomplished to generate gold surface-bound thiolates. However, in the non-base-promoted adsorptions, higher concentrations of the thioacetyl groups, relative to that of thiol groups, are required to achieve monolayer coverage in a given time interval. A thiol-terminated phenylene ethynylene system was shown to have a tilt angle of the long molecular axis of $<20^{\circ}$ from the normal to the substrate surface. These aromatic $\alpha, \omega$-dithiol-derived monolayers provide the basis for studies leading to the design of molecular wires capable of bridging proximate gold surfaces.

Charge transport in and the measurement of the conductance of single organic molecules is an intriguing, experimentally challenging, and long sought goal. These measurements have been performed on benzene-1,4dithiolate connected between stable proximal metallic gold contacts using a MCB [11]. The metal-molecule-metal configuration presents the molecular embodiment of a system analogous to a quantum dot, with the potential barriers replaced by the contact barrier of the gold-thiolate endgroups (figure 1). While we believe that we are measuring a single molecule, there is no way to be sure in this configuration since we do not have a third probe to image the interface. The results show a highly reproducible apparent Coulomb gap at about $0.7 \mathrm{~V}$ at room temperature. Further data are needed to confirm that this is, indeed, a Coulomb gap. The molecule provides a resistance of $22.2 \mathrm{M} \Omega$. A control experiment with tetrahydrofuran (THF) solvent alone exhibited a resistance of 1-2 G $\Omega$, independent of electrode spacing. When the solvent was evaporated, regular vacuum tunneling with a much higher resistance was observed. This study provides a direct, quantitative measurement of the molecular conductance of a junction containing a single molecule, a fundamental step in the emerging field of molecular scale electronics.

By measuring the conductivity of a series of molecules that are systematically altered, the contribution of each component of a molecular scale electronic device can be determined. For example, the simplest experiment of this type would be to measure the conductivity of 

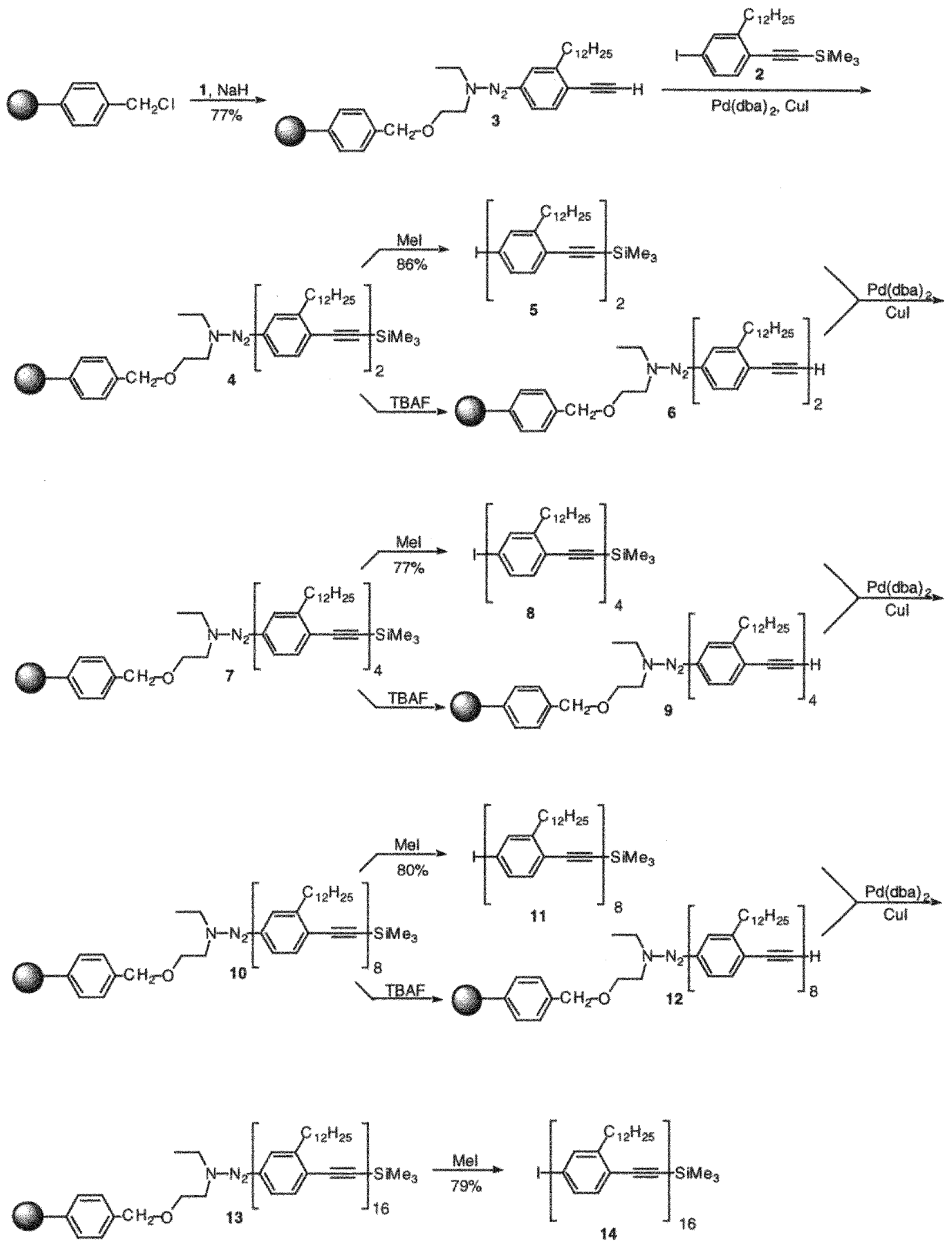

Scheme II. The polymer supported iterative divergent/convergent approach to the dodecyl-containing linear oligomers.

identical arrays of molecular wires in which only the wires' attachment to the gold surface is altered. Thus, by varying the molecular alligator clip and examining the molecules' conductivity, the contribution of the alligator clip to the resistance of the molecular wire can be determined. Our initial work has focused on varying the alligator clip by 


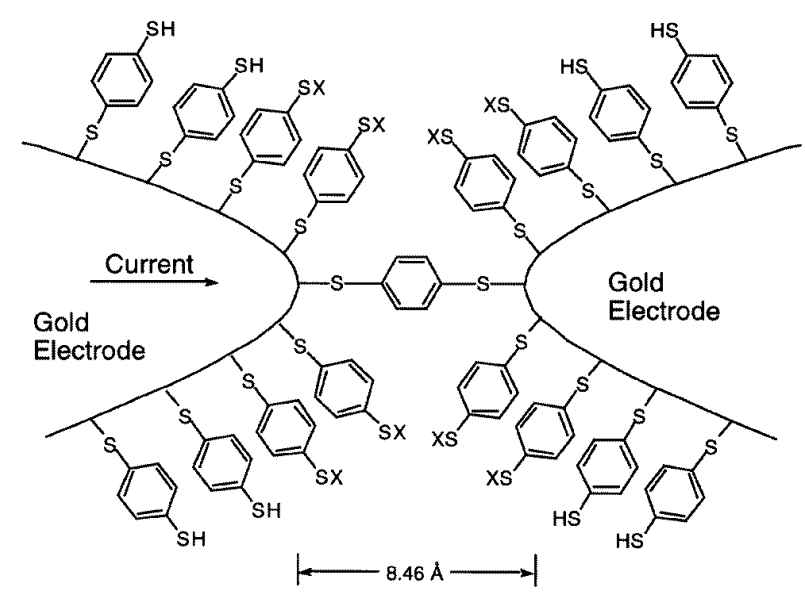

Figure 1. The desired placement of benzene-1, 4-dithiolate between gold electrodes in a MCB $(X=H, A u)$.

proceeding down the chalcogens from $\mathrm{S}$ to $\mathrm{Se}$ to Te.

We first desired a stable, yet easily removable, protecting group for selenium that would allow us to use the same in situ deprotection protocol we had employed for the thioacetates. An investigation of several protecting groups led us to conclude that, as with sulfur, the acetyl group was the protecting group of choice [12]. We have studied the molecular packing of both the S- and Se-biphenyl compounds and have found that they form close-packed, wellordered monolayers on $\mathrm{Au}$. Recently, molecular arrays of both 4, 4'-biphenyl thioacetate and 4, 4'-biphenyl selenoacetate have been subjected to conductivity examination using an evaporated-metal-top-contact/molecules/metallicbottom-contact configuration ( $X=S$, Se in figure 2$)$. The conduction in these systems is not due to metal insertion into the molecular arrays since these metallic shorts would have very low resistance. It appears that the selenium alligator clip creates a higher barrier for electron transport regardless of the direction of electron flow [13]. This is puzzling in light of the greater metallic character of Se versus $S$ which we reasonably assumed would yield a lower barrier for Se. This is also in disagreement with recent theoretical calculations which indicate that the barrier height for selenium should be significantly lower than for sulfur [14]. Additional work is underway to explain this unexpected result and examine the corresponding tellurium compound.

In summary, we have made four significant breakthroughs in the syntheses and testing of molecular scale electronic devices. First, we have completed the synthesis of the dodecyl-containing 16-mer on Merrifield's resin (chloromethyl polystyrene) using the iterative divergent/convergent approach; a method which significantly streamlines the preparation. Second, we have studied the formation of SAMs and multilayers on gold surfaces of rigid rod conjugated oligomers that have thiol, $\alpha, \omega$ dithiol, thioacetyl, or $\alpha, \omega$-dithioacetyl end groups. Third, molecules of benzene-1, 4-dithiol have been self-assembled onto the two facing gold electrodes of a MCB to form a statically stable gold-sulfur-aryl-sulfur-gold system, which allows for direct observation of charge transport through the molecules. Finally, we are investigating what effect vary-

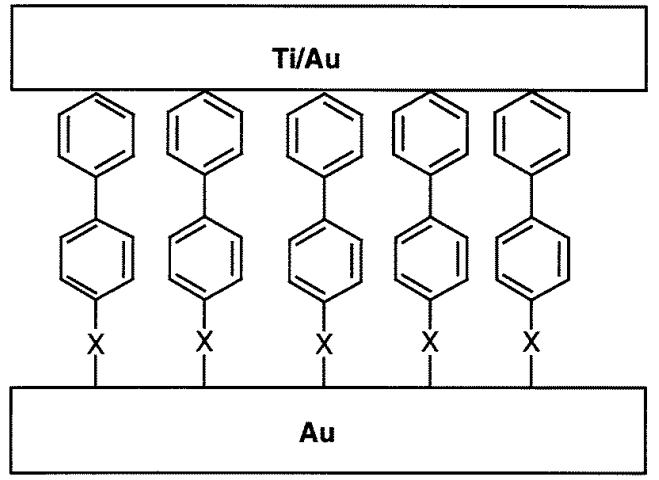

Figure 2. System for measuring conductivity of a molecular array $(\mathrm{X}=$ molecular alligator clip $\mathrm{S}$ or $\mathrm{Se})$.

ing the molecular alligator clip has on the molecule scale wire's conductivity [15].

\section{Acknowledgment}

We thank the Defense Advanced Research Projects Agency for support of this work.

\section{References}

[1] For some recent background work on molecular scale electronics, see

Aviram A (ed) 1992 Molecular Electronics: Science and Technology (Conf. Proc. 262) (New York: American Institute of Physics)

Miller J S 1990 Adv. Mater. 2378

Miller J S 1990 Adv. Mater. 2495

Miller J S 1990 Adv. Mater. 2601

Birge R R (ed) 1991 Molecular and Biomolecular Electronics (Advances in Chemistry Series 240) (American Chemical Society)

Kirk W P and Reed M A 1992 Nanostructures and Mesoscopic Systems (New York: Academic)

[2] For some theoretical considerations on molecular scale wires, see

Samanta M P, Tian W, Datta S, Henderson J I and Kubiak C P 1996 Phys. Rev. B 53 R7626

Mujica V, Kemp M, Roitberg A and Ratner M $1996 \mathrm{~J}$. Phys. Chem. 1047296

Joachim C and Vinuesa J F 1996 Europhys. Lett. 33635

[3] For some recent background work on the formation of molecular-based transporters and devices, see

Grosshenny V, Harriman A and Ziessel R 1995 Angew. Chem., Int. Ed. Engl. 341100

Langler L, Stockman L, Heremans J P, Bayor V, Olk C H, Van Haesendonck C, Bruynseraede Y and Issi J-P 1995 Synth. Met. 701393

Pascual J I, Méndez J, Gómez-Herrero J, Baró A M, Garcia N, Landman U, Luedtker W D Bogachek E N and Cheng H-P 1995 Science 2671793

Purcell S T, Garcia N, Binh V T, Jones L II and Tour J M 1994 J. Am. Chem. Soc. 11611985

Martin C R 1994 Science 2661961

Seth J, Palaniappan V, Johnson T E, Prathapan S, Lindsey J S and Bocian D F 1994 J. Am. Chem. Soc. 11610578 Gust D 1994 Nature 372133

Wu C and Bein T 1994 Science 2661013 
Wagner R W, Lindsey J S, Seth J, Palaniappan V and Bocain D F 1996 J. Am. Chem. Soc. 1183996

Sailor M J and Curtis C L 1994 Adv. Mater. 6688

Brigelletti F, Flamigni L, Balzani V, Collin J, Sauvage J, Sour A, Constable E C and Thompson A M W C 1994 J. Am. Chem. Soc. 1167692

Wu C and Bein T 1994 Science 2641757

Moerner W E 1994 Science 26546

Sessler J L, Capuano V L and Harriman A 1993 J. Am. Chem. Soc. 1154618

Farazdel A, Dupuis M, Clementi E and Aviram A $1990 \mathrm{~J}$. Am. Chem. Soc. 1124206

Tour J M, Wu R and Schumm J S 1991 J. Am. Chem. Soc. 1137064

Dai H, Wong E W and Lieber C M 1996 Science 272523

Wu R, Schumm J S, Pearson D L and Tour J M 1996 J. Org. Chem. 616906

Ward M D 1996 Chem. Ind. 569

[4] Petty M C, Bryce M R and Bloor D 1995 Introduction to Molecular Electronics ed M C Petty et al (New York: Oxford University Press)

[5] Skotheim T A (ed) 1986 Handbook of Conducting Polymers (New York: Dekker)

[6] Tour J M, Jones L II, Pearson D L, Lamba J S, Burgin T, Whitesides G W, Allara D L, Parikh A N and Atre S 1995 J. Am. Chem. Soc. 1179529
Dhirani A, Zehner R W, Hsung R P, Guyot-Sionnest P and Sita L R 1996 J. Am. Chem. Soc. 1183319

[7] Bumm L A, Arnold J J, Cygan M T, Dunbar T D, Burgin T P, Jones L II, Allara D L, Tour J M and Weiss P S 1996 Science 2711705

[8] Schumm J S, Pearson D L and Tour J M 1994 Angew. Chem., Int. Ed. Engl. 331360

Pearson D L, Schumm J S and Tour J M 1994 Macromolecules 272348

Pearson D L and Tour J M 1997 J. Org. Chem. 621376

[9] Jones L II, Schumm J S and Tour J M 1997 J. Org. Chem. 621388

[10] Young J K, Nelson J C and Moore J S 1994 J. Am. Chem. Soc. 11610841

Bharathi P, Patel U, Kawaguchi T, Pesak D J and Moore J S 1995 Macromolecules 285955

Hodge P and Sherrington D C (ed) 1980 Polymer-Supported Reactions in Organic Synthesis (New York: Wiley)

Nelson J C, Young J K and Moore J S 1996 J. Org. Chem. 618160

[11] Reed M A, Zhou C, Muller C J, Burgin T P and Tour J M 1997 Science 278252

[12] Reinerth W A and Tour J M 1998 J. Org. Chem. 632397

[13] Reed M A, Zhou C, Reinerth W A and Tour J M unpublished

[14] Tour J M and Seminario J unpublished 